\title{
Espécie nova de Pseudotyphistes (Araneae, Linyphiidae) do sul do Brasil
}

\author{
Everton Nei Lopes Rodrigues \& Ricardo Ott
}

Museu de Ciências Naturais, Fundação Zoobotânica do Rio Grande do Sul. Rua Dr. Salvador França, 1427, 90690-000 Porto Alegre, RS, Brasil. (enlrodrigues@yahoo.com.br; rott@fzb.rs.gov.br)

\begin{abstract}
New species of Pseudotyphistes (Araneae, Linyphiidae) from southern Brazil. Pseudotyphistes biriva sp. nov. from state of Rio Grande do Sul is described and illustrated. Ecological data of the new species are presented.
\end{abstract}

KEYWORDS. Erigoninae, Pseudotyphistes, Neotropics, taxonomy, ecology.

RESUMO. Pseudotyphistes biriva sp. nov. do Estado do Rio Grande do Sul é descrita e ilustrada. Dados ecológicos da nova espécie são apresentados.

PALAVRAS-CHAVE. Erigoninae, Pseudotyphistes, Região Neotropical, taxonomia, ecologia.

O gênero Pseudotyphistes foi proposto por Brignoli (1972) que designou como espécie-tipo Pseudotyphistes pennatus Brignoli, 1972, descrita para o Uruguai, coletada em uma caverna. Foi diagnosticado, principalmente, pela forma do címbio do palpo do macho (BRIGNOLI, 1972).

Neste trabalho é descrita uma nova espécie de Pseudotyphistes coletada em serapilheira e são apresentados dados de ecologia a partir de indivíduos obtidos com armadilhas de solo ("pitfall-traps").

\section{MATERIAL E MÉTODOS}

Os exemplares foram coletados no Centro de Pesquisas e Conservação da Natureza Pró-Mata, São Francisco de Paula, RS. Seis áreas de floresta foram escolhidas para a realização do trabalho: duas de Floresta Ombrófila Mista, bem conservadas (ARA); duas de floresta secundária (SEC) e duas com silvicultura de Pinus spp. (PIN). Em cada área foram escolhidos dois transectos e, sobre cada um, instaladas cinco armadilhas de solo, uma a cada 10 metros, num total de dez armadilhas para cada uma das áreas. As amostragens foram realizadas, ininterruptamente, nos mesmos pontos ao longo de 20 meses, totalizando 18 datas amostrais entre 25.X.2000 e 18.V.2002.

Para comparação do número de indivíduos registrados em cada sexo, foi utilizado o teste de "Wilcoxon" para dados que não seguem distribuição normal ("Wilcoxon Signed Rank Test" - WSRT) $\operatorname{com} \alpha=$ 0,05 (ZAR, 1999); os dados foram agrupados considerandose a soma total de indivíduos de cada sexo, em cada uma das armadilhas (10 em cada área), ao longo de todo período amostral (20 pares). Cálculos foram realizados com o auxílio do programa estatístico SigmaStat (JANDEL, 1995).

O material está depositado na coleção aracnológica do Museu de Ciências Naturais, Fundação Zoobotânica do Rio Grande do Sul (MCN; E. H. Buckup) e Museu de Ciências e Tecnologia da Pontifícia Universidade Católica do Rio Grande do Sul (MCTP; A. A. Lise), ambos em Porto Alegre, RS, Brasil. A posição do tricobótrio no metatarso da perna I (TmI) foi calculada conforme Millidge (1980). A terminologia das descrições segue Millidge $(1985,1991)$. Para o estudo das estruturas internas da genitália da fêmea, o epígino foi submerso em ácido lático por aproximadamente 30 minutos. Medidas são dadas em milímetros $(\mathrm{mm})$ e as variações apresentadas a partir de medidas realizadas em 10 machos e 10 fêmeas.

\section{Pseudotyphistes biriva sp. nov. (Figs. 1-8)}

Material-tipo. Holótipo ơ', BRASIL, Rio Grande do Sul: São Francisco de Paula (Centro de Pesquisas e Conservação da Natureza Pró-Mata), 01.V.2001, R. Ott col., coletado com armadilha de solo (pitfall-trap) (MCN 40701). Parátipos: mesma localidade e data do holótipo, o (MCN 40702); O’, †, 07.IV.2001

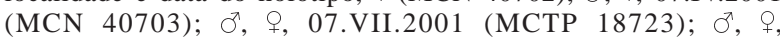
03.III.2001 (MCTP 18724), todos coletados com armadilhas de solo por R. Ott.

Etimologia. O nome específico em aposição referese aos habitantes de cima da serra, descendentes de bandeirantes, ou aos tropeiros paulistas, os quais geralmente andavam em mulas e tinham um sotaque peculiar, diferente dos da fronteira do Rio Grande do Sul com o Uruguai.

Diagnose. O palpo do macho de Pseudotyphistes biriva sp. nov. assemelha-se ao de P. pennatus Brignoli, 1972 (BRIGNOLI, 1972: figs. 15,18) por apresentar em comum uma macroseta dorsal no címbio (Fig. 2); difere pela forma da macroseta, que em $P$. biriva é retorcida, êmbolo bem mais longo e sinuoso, projetando-se até o ápice do címbio (Figs. 2-4), paracímbio longo sobre o subtégulo (Fig. 2), apófise tibial retrolateral (Figs. 2, 5) com região distal mais curva do que em P. pennatus. Fêmea com epígino similar ao de P. pennatus Brignoli, 1972 (Brignoli, 1972: figs. 16-17) por compartilhar a base alargada e por 


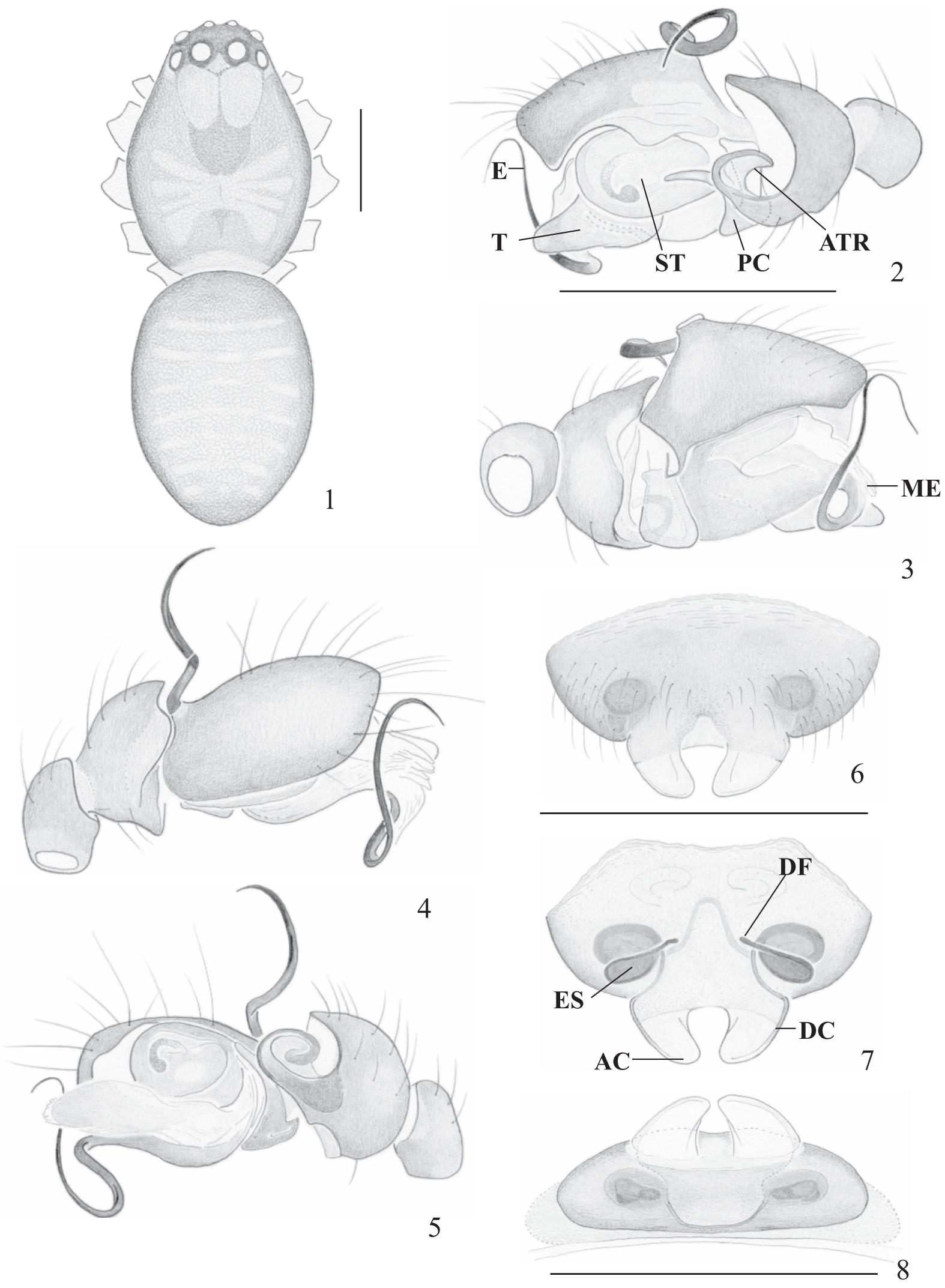

Figs. 1-8. Pseudotyphistes biriva sp. nov. 1-5, macho: 1, dorsal. 2-5, palpo: 2, ectal; 3, mesal; 4, dorsal; 5, ventral. 6-8, epígino: 6, ventral; 7, dorsal, clarificado; 8, posterior. (AC, abertura de copulação; ATR, apófise tibial retrolateral; DC, ducto de copulação; DF, ducto de fertilização; E, êmbolo; ES, espermateca; ME, membrana embólica; PC, paracímbio; ST, subtégulo; T, tégulo). Barras: 0,3 mm. 
projetar-se posteriormente e pela trajetória dos ductos de copulação nas laterais da placa do epígino; distinguese pelas espermatecas e ductos de fertilização serem mais separados entre si e pela porção distal do epígino bifurcada (Figs. 6-8).

Descrição, holótipo ơ. Comprimento total 1,52. Carapaça, comprimento 0,76, largura 0,58, altura 0,36. Clípeo, altura 0,16. Esterno, comprimento 0,42, largura 0,37. Abdômen, comprimento 0,81 , largura 0,52 , altura 0,56 . Fórmula das pernas $1 / 4 / 2 / 3$. Comprimento $1 / 2 / 3 / 4$ : fêmures 0,61/0,51/0,42/0,60; patelas 0,18/0,20/0,18/0,18; tíbias $0,53 /$ $0,43 / 0,35 / 0,52$; metatarsos $0,40 / 0,37 / 0,32 / 0,40$; tarsos $0,31 /$ 0,27/0,25/0,23. Total 2,03/1,78/1,52/1,93. Posição do tricobótrio no metatarso da perna I (TmI) 0,42. Metatarsos com tricobótrios, exceto IV. Carapaça (Fig. 1) castanhoavermelhada com bordas denegridas e detalhes castanhoescuros; área ocular enegrecida, mais larga do que longa. Largura da fila ocular anterior 0,23 e posterior 0,26 . OLA e OMP maiores que os demais e OMA menos desenvolvidos. Esterno castanho-escuro com bordas negras; enditos e quelíceras castanho-amarelados. Perna I com fêmur amarelo-avermelhado e demais com todos artículos amarelo-claros. Abdômen dorsalmente cinzaescuro com pontos despigmentados. Ventre cinza com área em torno das fiandeiras cinza-escuro. Címbio alto, com macroseta dorsal longa e sinuosa; presença de membrana embólica; paracímbio longo sobre o subtégulo e tégulo projetado distalmente (Figs. 2-5).

Parátipo 9 (MCN 40702). Comprimento total 1,54. Carapaça, comprimento 0,69, largura 0,52, altura 0,27. Clípeo, altura 0,12. Esterno, comprimento 0,41, largura 0,36. Abdômen, comprimento 0,98, largura 0,75, altura 0,70. Fórmula das pernas $1 / 4 / 2 / 3$. Comprimento $1 / 2 / 3 / 4$ : fêmures $0,51 / 0,46 / 0,41 / 0,51$; patelas $0,18 / 0,18 / 0,18 / 0,18$; tíbias $0,46 /$ $0,37 / 0,30 / 0,44$; metatarsos $0,35 / 0,31 / 0,26 / 0,33$; tarsos $0,28 /$ 0,25/0,20/0,25. Total 1,78/1,57/1,35/1,71. Posição do tricobótrio no metatarso da perna I (TmI) 0,40. Todos os metatarsos com tricobótrios, exceto o IV. Carapaça castanho-escura com bordas negras; área ocular enegrecida, mais larga do que longa. Largura da fila ocular

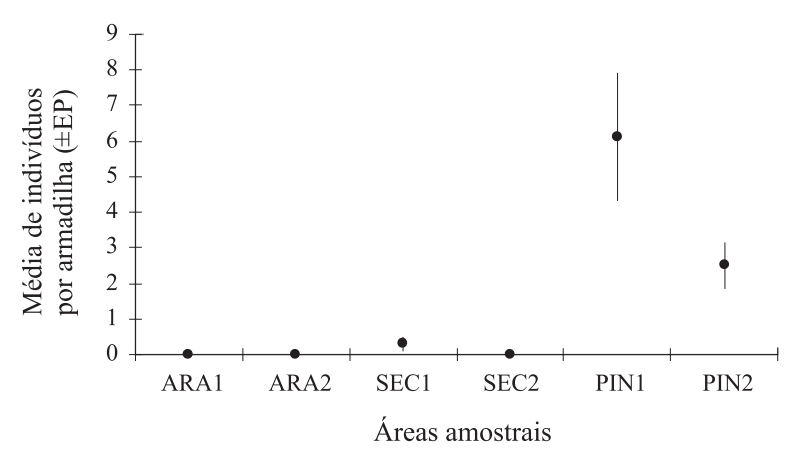

Fig. 9. Médias \pm Erro Padrão (EP) de indivíduos de Pseudotyphistes biriva sp. nov. capturados por armadilha nas áreas amostrais, no período de 25.X.2000 a 18.V.2002, no CPCN Pró-Mata, São Francisco de Paula, RS, Brasil (ARA, Floresta Ombrófila Mista; SEC, floresta secundária; PIN, silvicultura de Pinus spp.). anterior 0,21 e posterior 0,26. OLA e OMP maiores que os demais e OMA menos desenvolvidos. Esterno castanhoescuro; enditos e quelíceras castanhos. Pernas amareloclaras. Abdômen dorsalmente castanho-amarelado. Ventre castanho-amarelado e castanho-escuro em torno das fiandeiras. Epígino com ductos de copulação que seguem um percurso lateral à projeção posterior da placa; aberturas copulatórias dorsalmente na área posterior das bordas da placa do epígino e ductos de fertilização na região mediana com origem nas espermatecas (Figs. 6-8).

Comprimento total: machos 1,46-1,60; fêmeas 1,541,66 .

Material examinado. BRASIL, Rio Grande do Sul: São Francisco de Paula (Centro de Pesquisas e Conservação da Natureza Pró-Mata), 9 ơ', 16 ९, 25.X.2000-01.V.2001, R. Ott col., pitfall trap (MCN 39385-39388); $34 \sigma^{\top}, 4$ +, $25 . X .2000-$ 18.V.2002, mesma localidade e coletor (MCTP 18725-18751).

\section{Distribuição. Rio Grande do Sul.}

Ecologia. No período entre 25.X.2000 e 18.V.2002 foram amostrados 89 espécimes adultos - 46 machos e 43 fêmeas. Segundo o "teste de Wilcoxon", a diferença no número de machos e fêmeas capturados em cada armadilha não foi significativamente diferente (WSRT; $P$ $=0,528)$.

A espécie esteve presente, quase que exclusivamente, nas áreas com silvicultura de Pinus spp., estando totalmente ausente nas amostragens realizadas nas áreas de Floresta Ombrófila Mista bem-conservada e representada por apenas três indivíduos em uma das áreas secundárias (Fig. 9). O motivo para a abundância em silvicultura de Pinus não está claro, mas, como a espécie foi registrada com baixas abundâncias, ou até mesmo inexistente em florestas secundárias, o mais provável é que o tipo de serapilheira ou o microclima das áreas com silvicultura tragam vantagens para a espécie.

No fenograma (Fig. 10) visualiza-se um abrupto decréscimo na atividade das fêmeas, imediatamente após o início das amostragens, o que possivelmente possa

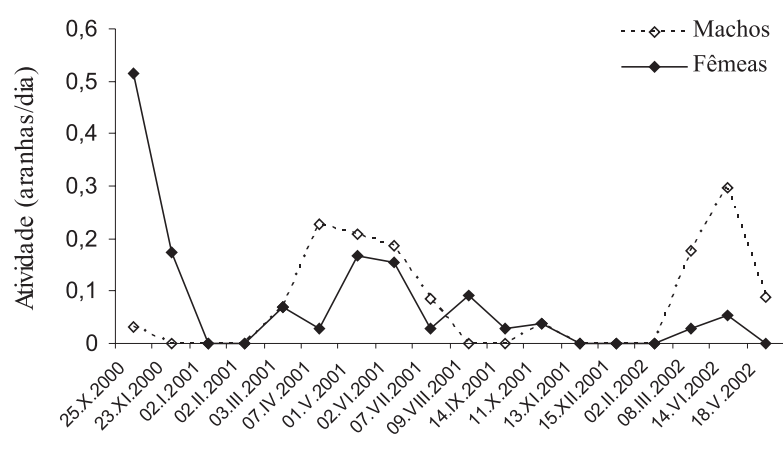

Datas amostrais

Fig. 10. Fenograma representando a atividade (total de aranhas capturadas/dias de amostragem) de machos e fêmeas de Pseudotyphistes biriva sp. nov., em cada data amostral, ao longo do período de coletas (25.X.2000 a 18.V.2002), no CPCN PróMata, São Francisco de Paula, RS, Brasil. 
estar relacionado com um efeito inicial, considerável, de remoção de indivíduos pela ação das armadilhas de solo terem sido postas nos mesmos locais. Todavia, machos e fêmeas aparentam ter sua atividade aumentada durante $o$ período de outono, sendo que para os machos, torna-se evidente um aumento das atividades durante as coletas de 2002. A espécie parece apresentar um padrão de sazonalidade univoltino, com grande atividade na estação do outono. Espécies de Linyphiidae com períodos de grande atividade exclusivamente no outono parecem ser pouco comuns na América do Norte (Aitchinson, 1984; DRANEY, 1997; Bolduc et al., 2005). Segundo HuHTA (1965) aumentos na abundância de determinadas espécies durante o outono, em determinados ecossistemas florestais, poderiam estar associados ao microclima mais úmido deste período em relação ao verão.

Agradecimentos. À curadora da coleção aracnológica do $\mathrm{MCN}$, pelo acesso ao material e à bibliografia. Ao curador do MCTP, pelo acesso à coleção. À Maria Aparecida de L. Marques $(\mathrm{MCN})$ e aos revisores pelas sugestões apresentadas. À Capes pelo auxílio financeiro e à PUCRS pela utilização da área do CPCN Pró-Mata, durante o período de realização da tese de doutorado do segundo autor.

\section{REFERÊNCIAS BIBLIOGRÁFICAS}

Aitchinson, C. W. 1984. The phenology of winter-active spiders. The Journal of Arachnology 12:249-271.

Bolduc, E.; Buddle, C. M.; Bostanian, N. J. \& Vincent, C. 2005. Ground-dwelling spider fauna (Araneae) of two vineyards in southern Quebec. Community and Ecosystem Ecology 34(3):635-645.

BrignOLI, P. M. 1972. Sur quelques araignées cavernicoles d'Argentine, Uruguay, Brésil et Venezuela récoltées par le D P. Strinati (Arachnida, Araneae). Revue Suisse de Zoologie 79:361-385.

Draney, M. L. 1997. Ground-layer spiders (Araneae) of a Georgia Piedmont floodplain agroecosystem: species list, phenology and habitat selection. The Journal of Arachnology 25:333-351.

Huhta, V. 1965. Ecology of spiders in the soil and litter of Finnish forests. Annales Zoologici Fennici 2(4):260-308.

JANDEL. 1995. SigmaStat statistical user's manual, version 2.0. Jandel, San Rafael.

Millidge, A. F. 1980. The erigonine spiders of North America. Part 1. Introduction and taxonomic background (Araneae: Linyphiidae). The Journal of Arachnology 8:97-107.

1985. Some linyphiid spiders from South America (Araneae, Linyphiidae). American Museum Novitates 2836:1-78. 1991. Further linyphiid spiders (Araneae) from South America. Bulletin of the American Museum of Natural History 205:1-199.

ZAR, J. H. 1999. Biostatistical analysis. 4 ed. New Jersey, Prentice-Hall. 663p. 